\title{
Familial Mediterranean fever association with schizophrenia: insights from big data analysis
}

Kassem Sharif ${ }^{1,2,3}$, Abdulla Watad ${ }^{2,3}$, Nicola Luigi Bragazzi ${ }^{4}$, Yarden Yavne ${ }^{2,3}$, Charlie Bridgewood ${ }^{1}$, Dennis McGonagle ${ }^{1}$, Doron Comanhester ${ }^{5}$, Arnon D. Cohen ${ }^{5}$, Howard Amital',3

\author{
${ }^{1}$ University of Leeds, United Kingdom \\ ${ }^{2}$ Sheba Medical Centre, Israel \\ ${ }^{3}$ Sackler Faculty of Medicine, Tel Aviv University, Tel Aviv, Israel \\ ${ }^{4}$ University of Genoa, Italy \\ ${ }^{5}$ Clalit Health Services, Israel
}

Submitted: 15 August 2019

Accepted: 26 February 2020

Arch Med Sci

DOI: https://doi.org/10.5114/aoms.2020.95463

Copyright (c) 2020 Termedia \& Banach

\section{Corresponding author:}

Kassem Sharif

Sheba Medical Centre,

Tel Hashomer, Israel

E-mail: Kassemsharif@gmail. com

\begin{abstract}
Introduction: Several autoimmune diseases have been associated with schizophrenia; however, little is known about putative links with auto-inflammatory conditions. We investigated the association between familial Mediterranean fever (FMF), a paradigmatic auto-inflammatory disease, and schizophrenia and assessed the impact of the latter on the survival of FMF patients.

Material and methods: A case-control study was performed by utilising the database of Clalit Health Services, the largest health maintenance organisation in Israel. Familial Mediterranean fever patients were compared to age- and sex-matched controls in terms of prevalence of schizophrenia. The $\chi^{2}$ test was used to assess the distribution of categorical variables, while the t-test was applied for continuous variables. Analysis regarding survival was performed using Kaplan-Meier curves, log rank test, and multivariate Cox proportional-hazards method. Statistical analysis was performed using SPSS version 24.0, IBM, USA.

Results: The study included 7747 FMF patients and 10,080 age- and sexmatched controls (case-control match $1: 1.3$ ). On univariate analysis, 50 FMF patients $(0.6 \%)$ and 89 controls $(0.9 \%)$ had schizophrenia. On multiple logistic regression model, FMF was inversely associated with schizophrenia $(\mathrm{OR}=0.64$ [95\% Cl: 0.43-0.90], $p=0.0173)$. Familial Mediterranean fever $(H R=1.43$ [95\% Cl: 1.23-1.66]) and schizophrenia ( $\mathrm{HR}=3.97$ [95\% Cl: 1.47-10.70]) were independently associated with all-cause mortality. However, schizophrenia as a co-morbidity in FMF subjects did not worsen their prognosis $(\mathrm{HR}=2.17$ [95\% Cl: 0.60-7.86]).

Conclusions: Familial Mediterranean fever patients have a significantly lower proportion of schizophrenia than controls. The current evidence shows that auto-inflammatory conditions confer protection against schizophrenia development. Patients with either FMF or schizophrenia are at higher risk of all-cause mortality, a finding that calls for assessment of better medical management of mortality outcomes.
\end{abstract}

Key words: schizophrenia, epidemiology, familial Mediterranean fever (FMF), autoinflammation.

\section{Introduction}

Familial Mediterranean fever (FMF) is a common hereditary, autoinflammatory disease, which is significantly associated with several ethni- 
cities in the Mediterranean region, chiefly Turkish, Jewish, and Arabic communities [1].

Familial Mediterranean fever is an autosomal recessive disease that results from a point mutation in the Mediterranean fever gene (MEFV), located on the short arm of chromosome 16 [2]. Various mutations of the MEFV gene exist, and genotype-phenotype correlation studies have demonstrated a more severe disease course in individuals who are homozygous for the M694V mutation [3].

Schizophrenia is a chronic psychiatric disorder characterised by a heterogeneous presentation of negative and positive symptoms, which results in disruption of patients' thoughts and emotions and interferes with the individuals' capacity to engage in social events and to develop meaningful relationships [4]. Studies of neurological manifestations in paediatric FMF patients have found a significant association with various neurological disorders such as headache, epilepsy, pseudotumor cerebri, tremor, and multiple sclerosis, although several of these conditions are relatively rare [5].

Familial Mediterranean fever patients suffer from greater emotional stress in comparison to healthy patients, thus further adding to the morbidity of this patient population. Recent data indicate that FMF patients have considerably higher risk of developing depression and anxiety, which adversely affects patients' quality of life [5-7]. Indeed, disease management using interleukin (IL)-1 antagonists led to significant improvement in physical function, role limitation due to emotional problems, emotional well-being, social functioning, and quality of life [8].

Clinical overlaps exist between these conditions. For instance, Eaton et al. [9] investigated the co-occurrence of autoimmune conditions with schizophrenia and demonstrated an increased occurrence of certain autoimmune conditions amongst schizophrenic patients, thus suggesting a shared genetic diathesis. Moreover, genome- wide association studies (GWAS) have identified several single nucleotide polymorphisms (SNPS) in schizophrenia that are primarily implicated in immune and inflammatory response [10].

However, the relationship between FMF and schizophrenia remains to be elucidated. In our current investigation, we aimed to assess the association between these disorders in a real-life population by utilising the Clalit Health Services (CHS) database, the largest Health Maintenance Organisation (HMO) in Israel.

\section{Material and methods}

This study was designed as a case-control study based on the chronic disease registry of the CHS, the largest integrated health service in Israel, which provides healthcare to more than $4,400,000$ insured enrolees (roughly 50\% of the Israeli population).

Data presented in the CHS database undergo a series of verification processes, which includes comparison of diagnoses from various sources. The validity of the data was verified in previously published studies [11, 12]. Wide-scale epidemiological studies can be conducted in real time on heterogeneous groups by using data-mining techniques. Familial Mediterranean fever patients were identified as patients with at least one diagnosis of FMF in their medical records, either provided by a general practitioner, a primary care physician, or a specialist. Familial Mediterranean fever has ethnic prediction to nations of the Mediterranean region. CHS entails a large database of FMF patients including approximately 7700 patients. The control group consisted of randomly selected CHS enrolees, with the exclusion of patients with an established diagnosis of FMF. Controls were age- and sex-matched to cases. Similarly, the diagnosis of schizophrenia included patients with these diagnoses in their medical records as entered by specialists in the CHS registry.

Additional data collected from the CHS database included age, gender, socioeconomic status (SES), body mass index (BMI), and smoking status. SES was defined according to the poverty index of the member's residence area. The poverty in dex is computed from several parameters including household income, education, as well as other factors. The composite index ranged from 1 to 20, with 1 as the lowest SES.

The study was approved by the Ethical Committee of CHS, located at the Soroka Medical Centre, Beer-Sheva, Israel.

\section{Statistical analysis}

The occurrence of schizophrenia and was compared between FMF patients and controls in the study sample. The $\chi^{2}$ test was used to assess the distribution of categorical variables, while the $t$-test and one-way analysis of variance (ANOVA) were applied for continuous variables. Moreover, the association between FMF and schizophrenia was assessed using a multivariate logistic regression model controlling for possible confounding factors.

Analyses regarding survival were performed using Kaplan-Meier curves, the log-rank test, and the multivariate Cox proportional-hazards method to detect factors associated with increased all-cause risk mortality, with adjustment for risk factors where appropriate.

Statistical analysis was performed using the commercial software Statistical Package for Social Sciences for Windows (SPSS version 24.0, IBM, USA). Figures with a $p$-value less than 0.05 were considered statistically significant. 


\section{Results}

Baseline characteristics of the study population and prevalence of schizophrenia

The present study included a total of 17,827 subjects, of whom 7747 were FMF patients and 10,080 were age- and sex-matched controls (case-control match $1: 1.3)$. The average age of the population was 38.4 years, and $50.5 \%$ of the total population were females. No significant difference was demonstrated with regard to age, gender, and BMI between the case and control groups. Two differences of note between the case and control groups was the higher rates of smoking and of SES amongst the FMF group. Fifty FMF patients $(0.6 \%)$ also had a diagnosis of schizophrenia, whereas amongst the healthy population, 89 patients $(0.9 \%)$ had schizophrenia. The variance in schizophrenia between the case and control group was considered non-significant. The mortality rate among the whole population was $4.0 \%: 3.4 \%$ in the control group and $4.7 \%$ in the case group ( $p<0.001$ ). The basic characteristics of the study population are further detailed in Table I.

\section{Independent predictors of schizophrenia}

On the multiple logistic regression model, FMF $(\mathrm{OR}=0.64,95 \% \mathrm{Cl}: 0.44-0.92, p=0.0173)$, age $(\mathrm{OR}=1.02,95 \% \mathrm{Cl}: 1.01-1.03, p=0.0010)$, and smoking $(\mathrm{OR}=2.84,95 \% \mathrm{Cl}: 1.69-3.63), p<0.0001)$ were all independently associated with schizophrenia. Additional details are included in Table II.

On the multivariate Cox proportional-hazards regression model (Table III), FMF diagnosis $(H R=0.62,0.43-0.90, p=0.0126)$ and smoking

Table I. Overall population, familiar Mediterranean fever patients (cases), and age-and-sex matched controls basic characteristics

\begin{tabular}{|lcccc|}
\hline Characteristic & $\begin{array}{c}\text { All population } \\
(N=17,827)\end{array}$ & $\begin{array}{c}\text { Controls without } \\
\text { FMF }(n=10,080)\end{array}$ & $\begin{array}{c}\text { FMF patients } \\
(n=7747)\end{array}$ & $\begin{array}{c}\text { Statistical significance } \\
(P \text {-value })\end{array}$ \\
\hline Age [mean \pm SD] & $38.43 \pm 19.62$ & $37.69 \pm 19.55$ & $39.38 \pm 19.68$ & NS \\
\hline Age at diagnosis [mean \pm SD] & $26.41 \pm 18.41$ & $25.67 \pm 18.35$ & $27.37 \pm 18.45$ & NS \\
\hline Gender, female, $n(\%)$ & $9000(50.5)$ & $5121(50.8)$ & $3879(50.1)$ & NS \\
\hline BMI [mean \pm SD] & $24.81 \pm 63.91$ & $24.42 \pm 50.61$ & $25.30 \pm 77.41$ & NS \\
\hline SES, $n(\%)^{a}$ & & & & $0.0054(0.0200$ for trend $)$ \\
\hline Low & $8370(50.6)$ & $4729(50.3)$ & $3641(51.1)$ & \\
\hline Medium & $5609(33.9)$ & $3153(33.5)$ & $2455(34.5)$ & \\
\hline High & $2548(15.4)$ & $1524(16.2)$ & $1024(14.4)$ & $<0.001$ \\
\hline Smoking, $n$ (\%) & $5000(28.0)$ & $2588(25.7)$ & $2412(31.1)$ & NS \\
\hline Schizophrenia, $n(\%)$ & $139(0.8)$ & $89(0.9)$ & $50(0.6)$ & $<0.001$ \\
\hline All-cause mortality, $n(\%)$ & $707(4.0)$ & $341(3.4)$ & $366(4.7)$ & \\
\hline
\end{tabular}

${ }^{a}$ Data available for $92.7 \%$ of the population. BMI - body mass index, FMF-familiar Mediterranean fever, SES - socio-economic status.

Table II. Multivariate logistic regression assessing covariates associated with schizophrenia

\begin{tabular}{|c|c|c|c|c|c|c|}
\hline Variable & Coefficient & Std. error & Wald & $P$ & OR & $95 \% \mathrm{Cl}$ \\
\hline \multicolumn{7}{|c|}{ Schizophrenia } \\
\hline Age & 0.02 & 0.00 & 10.75 & 0.0010 & 1.02 & $1.01-1.03$ \\
\hline Sex, female & -0.28 & 0.19 & 2.16 & 0.1421 & 0.76 & $0.52-1.10$ \\
\hline FMF & -0.45 & 0.19 & 5.67 & 0.0173 & 0.64 & $0.44-0.92$ \\
\hline SES (medium) & 0.26 & 0.20 & 1.66 & 0.1974 & 1.29 & $0.87-1.91$ \\
\hline SES (high) & 0.13 & 0.27 & 0.22 & 0.6374 & 1.13 & $0.67-1.91$ \\
\hline Smoking & 0.91 & 0.19 & 21.83 & $<0.0001$ & 2.48 & $1.69-3.63$ \\
\hline BMI [kg/m²] & 0.00 & 0.00 & 0.01 & 0.9136 & 1.00 & $1.00-1.00$ \\
\hline Constant & -5.71 & 0.28 & 428.26 & $<0.0001$ & & \\
\hline
\end{tabular}

BMI - body mass index, FMF - familiar Mediterranean fever, SES - socio-economic status. 
K. Sharif, A. Watad, N.L. Bragazzi, Y. Yavne, Ch. Bridgewood, D. McGonagle, D. Comanhester, A.D. Cohen, H. Amital

Table III. Multivariate Cox proportional-hazards regression assessing covariates associated with schizophrenia

\begin{tabular}{|lcccccc|}
\hline Covariate & b & SE & Wald & $P$ & HR & 95\% Cl \\
\hline & & \multicolumn{3}{c|}{ Schizophrenia } & & \\
\hline Age & 0.00 & 0.01 & 0.00 & 0.9800 & 1.00 & $0.99-1.01$ \\
\hline Sex, female & -0.30 & 0.19 & 2.58 & 0.1081 & 0.74 & $0.51-1.07$ \\
\hline FMF & -0.47 & 0.19 & 6.22 & 0.0126 & 0.62 & $0.43-0.90$ \\
\hline SES (medium) & 0.23 & 0.20 & 1.29 & 0.2562 & 1.25 & $0.85-1.85$ \\
\hline SES (high) & 0.20 & 0.26 & 0.56 & 0.4538 & 1.22 & $0.73-2.05$ \\
\hline Smoking & 0.79 & 0.19 & 17.20 & $<0.0001$ & 2.20 & $1.52-3.20$ \\
\hline BMI [kg/m ${ }^{2}$ ] & -0.00 & 0.00 & 0.00 & 0.9543 & 1.00 & $1.00-1.00$ \\
\hline
\end{tabular}

BMI - body mass index, FMF - familiar Mediterranean fever, SES - socio-economic status.

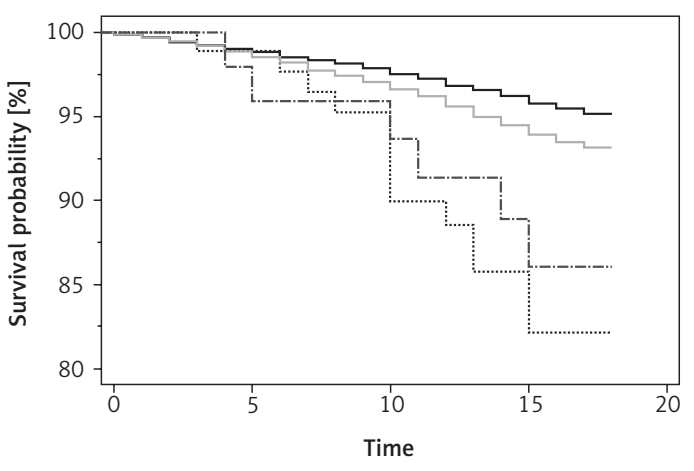

Controls without schizophrenia

........ Controls with schizophrenia

— FMF without schizophrenia

-.-. FMF with schizophrenia

Figure 1. Kaplan-Meier survival plot of study cohorts (namely, familiar Mediterranean fever [FMF] cases with and without schizophrenia, and controls without FMF with and without schizophrenia)

status $(\mathrm{HR}=2.20,95 \% \mathrm{Cl}: 1.52-3.20, p<0.0001)$ were predictors of schizophrenia.

\section{Survival analysis}

In the log-rank test, the Kaplan-Meier test results were statistically significant $\left(\chi^{2}=48.30\right.$, degrees of freedom $=3, p<0.0001$; Figure $1 \mathrm{~A})$, re- flecting the impact of a schizophrenia diagnosis on FMF and non-FMF patients' survival. In our analysis (Table IV), FMF patients had an increased all-cause mortality, as demonstrated when comparing FMF patients without schizophrenia to controls without schizophrenia ( $\mathrm{HR}=1.43,95 \% \mathrm{Cl}$ : $1.23-1.66)$. Among the control group, schizophrenia was shown to significantly increase the HR as compared to patients without schizophrenia $(\mathrm{HR}=3.97,95 \% \mathrm{Cl}$ : 1.47-10.70). However, schizophrenia as a co-morbidity did not worsen the survival of FMF patients $(\mathrm{HR}=2.17,95 \% \mathrm{Cl}: 0.60-7.86])$.

\section{Independent predictors of mortality}

In the Cox multivariate survival analysis (Table $\mathrm{V})$, older age $(\mathrm{HR}=1.08,95 \% \mathrm{Cl}: 1.07-1.08$, $p<0.0001)$, FMF diagnosis $(\mathrm{HR}=1.36,95 \% \mathrm{Cl}: 1.14-$ $1.63, p=0.0006)$, and schizophrenia ( $\mathrm{HR}=2.16$, $95 \% \mathrm{Cl}: 1.24-3.76, p=0.0063)$ were predictors of all-cause mortality. By contrast, female sex $(\mathrm{HR}=0.73,95 \% \mathrm{Cl}: 0.61-0.88, p=0.0010)$ and high SES (HR $=0.0223,95 \% \mathrm{Cl}: 0.56-0.96, p=0.0223)$ were independently associated with better survival.

\section{Discussion}

To the best of our knowledge, this is the first study to demonstrate a lower rate of schizophre-

Table IV. Hazard ratios (HRs) computed with their 95\% confidence interval (CI) obtained from the Kaplan-Meier survival analysis

\begin{tabular}{|lcccc|}
\hline HR & $\begin{array}{c}\text { Controls without } \\
\text { schizophrenia }\end{array}$ & $\begin{array}{c}\text { Controls with } \\
\text { schizophrenia }\end{array}$ & $\begin{array}{c}\text { FMF without } \\
\text { schizophrenia }\end{array}$ & $\begin{array}{c}\text { FMF with } \\
\text { schizophrenia }\end{array}$ \\
\hline Controls without schizophrenia & - & 3.97 & 1.43 & 3.10 \\
& & $-1.47-10.70$ & $1.23-1.66$ & $0.86-11.19$ \\
\hline Controls with schizophrenia & 0.25 & -0.36 & 0.78 \\
& $0.09-0.68$ & $0.13-0.97$ & $0.15-3.93$ \\
\hline FMF without schizophrenia & 0.70 & 2.79 & - & 2.17 \\
& $0.61-0.81$ & $1.03-7.51$ & & $0.60-7.86$ \\
\hline FMF with schizophrenia & 0.32 & 1.28 & 0.46 & - \\
& $0.09-1.17$ & $0.25-6.46$ & $0.13-1.67$ & \\
\hline
\end{tabular}

FMF-familiar Mediterranean fever. 
Table V. Cox multivariate survival analysis assessing covariates statistically associated with all-cause mortality

\begin{tabular}{|lcccccc|}
\hline \multicolumn{1}{|c}{ Covariate } & b & SE & Wald & P & HR & 95\% Cl \\
\hline & & \multicolumn{3}{c}{ Schizophrenia } & & \\
\hline Age & 0.08 & 0.00 & 707.95 & $<0.0001$ & 1.08 & $1.07-1.08$ \\
\hline Sex, female & -0.31 & 0.09 & 10.88 & 0.0010 & 0.73 & $0.61-0.88$ \\
\hline FMF & 0.31 & 0.09 & 11.91 & 0.0006 & 1.36 & $1.14-1.63$ \\
\hline SES (medium) & -0.06 & 0.10 & 0.35 & 0.5544 & 0.94 & $0.78-1.14$ \\
\hline SES (high) & -0.31 & 0.14 & 5.22 & 0.0223 & 0.73 & $0.56-0.96$ \\
\hline Smoking & 0.09 & 0.09 & 0.97 & 0.3247 & 1.10 & $0.91-1.31$ \\
\hline BMI [kg/m ${ }^{2}$ ] & 0.00 & 0.00 & 0.40 & 0.5249 & 1.00 & $1.00-1.00$ \\
\hline Schizophrenia & 0.77 & 0.28 & 7.46 & 0.0063 & 2.16 & $1.24-3.76$ \\
\hline
\end{tabular}

BMI - body mass index, FMF - familiar Mediterranean fever, SES - socio-economic status.

nia in FMF patients in comparison with controls. Moreover, although schizophrenia and FMF were both found to be independent predictors of allcause mortality, schizophrenia did not appear to have a significant impact upon the survival of FMF patients.

The association of schizophrenia with other autoimmune diseases has been the focus of several studies in the past years. In a study performed on the Danish psychiatric register, Eaton et al. [9] found a $45 \%$ increase in the risk for schizophrenia for subjects with any autoimmune disease. Of the autoimmune diseases, five were more frequently associated with schizophrenia: thyrotoxicosis, celiac disease, acute haemolytic anaemia, interstitial cystitis, and Sjogren's syndrome [9]. Nevertheless, certain autoimmune diseases did not increase the prevalence of schizophrenia. In a population-based cohort study on the Swedish population register, a significant reduction in the risk for rheumatoid arthritis ( $\mathrm{HR}=0.69,95 \% \mathrm{Cl}: 0.59-0.80)$ and ankylosing spondylitis $(\mathrm{HR}=0.62,95 \% \mathrm{Cl}$ : 0.45-0.86]) were noted among schizophrenia patients [13]. Corroborating these findings, a recent meta-analysis, comprising 31 studies and pooling data from more than 25 million eligible individuals, demonstrated a positive association between certain non-neurological autoimmune disorders and psychosis but not with others. Thus Graves' disease was positively associated with psychosis $(\mathrm{OR}=1.33,95 \% \mathrm{Cl}: 1.03-1.72)$, as was psoriasis $(\mathrm{OR}=1.7,95 \% \mathrm{Cl}: 1.29-2.84)$; however, rheumatoid arthritis (OR $=0.65,95 \% \mathrm{Cl}: 0.50-0.84])$ and ankylosing spondylitis (OR $=0.72,95 \% \mathrm{Cl}$ : $0.54-0.98])$ were not [14].

The mechanism driving the observed underlying association between certain autoimmune diseases and schizophrenia has been hypothesised to be multi-factorial in nature, involving inflammation and genetic predisposition, amongst other factors.
Dysregulated inflammatory processes have been implicated in schizophrenia aetiology with various, albeit inconsistent findings with regard to cytokine levels as markers of schizophrenia [15-18). In schizophrenia, higher levels of various cytokines have been reported, including IL-6, TNF- $\alpha$, and IL-1 $\beta$ $[19,20]$. Cytokine levels have been associated with structural brain changes, including decreased hippocampal volume and pre-cortex thinning, which is further associated with psychosis development $[21,22]$. Finally, it is probable that schizophrenia shares a genetic diathesis with autoimmune diseases. HOPA (human opposite paired) gene on chromosome Xq13 encodes for T4 receptor, which when mutated has been linked to hypothyroidism and schizophrenia [23-25].

If schizophrenia has been previously associated with inflammation and autoimmune disorders, how could this explain the protective effect of an FMF diagnosis demonstrated in our study? As mentioned before, it is known that the genetic loss of pyrin in FMF results in enhanced IL-1 $\beta$ and IL-18 release, and thus excess inflammation. Another aspect of this inflammatory process can be attributed to the difference in the immune pathways activated in auto-inflammatory conditions, as compared with autoimmune pathophysiology. In the former, the innate immune system is activated, resulting in direct tissue inflammation; however, in the latter, the consequent activation of the adaptive immune system results in autoantibody and T-cell production [26]. It is plausible that this differential activation of the various arms of the immune system would partly explain the negative association between FMF and schizophrenia in our study results.

Exploring the genetic factors behind the occurrence of both FMF and schizophrenia could provide an avenue for understanding the decreased risk of disease occurrence. GWAS demonstrated a sig- 
nificant association of the major histocompatibility complex and SNPs in schizophrenia patients [27]. Recently, negative SNP genetic correlation was documented between schizophrenia and rheumatoid arthritis ( $p=0.036)$, a result that is consistent with the epidemiological data reported on the negative association between the two disease entities, suggesting the prominence of genetic factors in both of the diseases' aetiologies [28]. Moreover, the $\mathrm{MHC}$ I haplotype HLA-B*08, a structure reported to predispose to rheumatoid arthritis, has been shown to confer a decreased risk of schizophrenia [29]. Although FMF is a prototypical monogenic auto-inflammatory condition, polymorphisms in HLA loci have been reported to influence the genetic and phenotypic disease penetrance [30]. With regard to schizophrenia, it is probable that the differential effect of MHC polymorphism could confer protective effects in patients with FMF, a finding that needs to be confirmed with GWAS. Taken together, it seems that in conditions defined as pure autoimmune conditions (conditions associated with MHC class II) a positive association with schizophrenia is demonstrated [13]. In contrast, immunological conditions defined as mixed pattern disease (e.g. ankylosing spondylitis), polygenic auto inflammatory diseases (e.g. inflammatory arthritis presenting as rheumatoid arthritis), and monogenic auto-inflammatory diseases (e.g. FMF) offer a protective effect against schizophrenia. Our study is the first to demonstrate this relationship in auto-inflammatory conditions and corroborates the findings in the other immunological disease subgroups.

With regard to mortality, our analysis showed that schizophrenia increased all-cause mortality in comparison with controls. In a large meta-analysis on schizophrenia patients the median standardised mortality ratio for all persons for all-cause mortality was 2.58 , suggesting an increased mortality risk in comparison to the general community [31].

Furthermore, FMF also had an increased mortality rate in comparison with healthy counterparts, an association that was the focus of investigation in a nationwide study, which demonstrated increased mortality in FMF male $(\mathrm{HR}=1.71,95 \% \mathrm{Cl}$ : 1.06-2.75) and female $(\mathrm{HR}=2.48,95 \% \mathrm{Cl}: 1.03-$ 5.99) patients. According to the findings of this study, the primary cause of death was renal amyloidosis, which accounted for 35\% and 60\% of deaths in men and women, respectively [32].

Our study has multiple strengths, including analysis of data in a real-life setting, which subsequently mirrors existing currents in the population. Moreover, the CHS database has one of the largest samples of FMF patients worldwide. However, several limitations warrant consideration, namely the observational design and lack of information regarding the temporal relationship between the conditions, which consequently precludes the determination of a causal relationship between FMF and schizophrenia. However, the reliability of CHS registry diagnosis has been alluded to by several studies and undergoes several levels of verification [11, 12]. Furthermore, information regarding disease duration and medications used to treat the conditions was not accessible.

\section{Conclusions}

In conclusion, our analysis revealed a reduced prevalence of schizophrenia in patients with FMF, suggesting a possible protective effect. Diagnosis with either FMF or schizophrenia was associated with an independent prediction of mortality. It seems that pure autoimmune conditions are positively associated with schizophrenia, whereas auto-inflammatory conditions confer a protective effect against schizophrenia. A better understanding of the genetic predispositions of both schizophrenia and FMF may help shed light on the pathophysiology and eventually assist in identifying better management and treatment options for both diseases.

\section{Conflict of interest}

The authors declare no conflict of interest.

\section{References}

1. Sarı İ, Birlik M, Kasifoğlu T. Familial Mediterranean fever: an updated review. Eur J Rheumatol 2014; 1: 21-33.

2. Cantarini L, Rigante D, Brizi MG, et al. Clinical and biochemical landmarks in systemic autoinflammatory diseases. Ann Med 2012; 44: 664-73.

3. Booty MG, Chae JJ, Masters SL, et al. Familial Mediterranean fever with a single MEFV mutation: where is the second hit? Arthritis Rheum 2009; 60: 1851-61.

4. Patel KR, Cherian J, Gohil K, Atkinson D. Schizophrenia: overview and treatment options. Pharmacy and Therapeutics 2014; 39: 638-45.

5. Canpolat M, Gumus H, Gunduz Z, et al. Neurological manifestations in familial Mediterranean fever: results of 22 children from a reference center in Kayseri, an urban area in Central Anatolia, Turkey. Neuropediatrics 2017; 48: 79-85.

6. Giese A, Ornek A, Kilic L, et al. Anxiety and depression in adult patients with familial Mediterranean fever: a study comparing patients living in Germany and Turkey. Int J Rheum Dis 2017; 20: 2093-100.

7. Makay B, Emiroglu N, Unsal E. Depression and anxiety in children and adolescents with familial Mediterranean fever. Clin Rheumatol 2010; 29: 375-9.

8. Sargin G, Kose R, Senturk T. Anti-interleukin-1 treatment among patients with familial Mediterranean fever resistant to colchicine treatment. Retrospective analysis. Sao Paulo Med I 2019; 137: 39-44.

9. Eaton WW, Byrne M, Ewald H, et al. Association of schizophrenia and autoimmune diseases: linkage of Danish national registers. Am J Psychiatry 2006; 163: 521-8.

10. Chan MK, Cooper JD, Heilmann-Heimbach S, et al. Associations between SNPs and immune-related circulating proteins in schizophrenia. Sci Rep 2017; 7: 12586. 
11. Sharif K, Tiosano S, Watad A, et al. The link between schizophrenia and hypothyroidism: a population-based study. Immunol Res 2018; 66: 663-7.

12. Sharif K, Watad A, Tiosano S, et al. The link between COPD and ankylosing spondylitis: A population based study. Eur J Intern Med 2018; 53: 62-5.

13. Sellgren C, Frisell T, Lichtenstein P, Landèn M, Askling J. The association between schizophrenia and rheumatoid arthritis: a nationwide population-based Swedish study on intraindividual and familial risks. Schizophr Bull 2014; 40: 1552-9.

14. Cullen AE, Holmes S, Pollak TA, et al. Associations between non-neurological autoimmune disorders and psychosis: a meta-analysis. Biol Psychiatry 2019; 85: 35-48.

15. Dickerson F, Stallings C, Origoni A, et al. Inflammatory markers in recent onset psychosis and chronic schizophrenia. Schizophr Bull 2016; 42: 134-41.

16. Joseph J, Depp C, Martin AS, et al. Associations of high sensitivity C-reactive protein levels in schizophrenia and comparison groups. Schizophr Res 2015; 168: 456-60.

17. Dahan S, Bragazzi NL, Yogev A, et al. The relationship between serum cytokine levels and degree of psychosis in patients with schizophrenia. Psychiatry Res 2018; 268: 467-72.

18. Poniewierska-Baran A, Tokarz-Deptuła B, Deptuła W. The role of innate lymphoid cells in selected disease states - cancer formation, metabolic disorder and inflammation. Arch Med Sci 2019; doi: https://doi. org/10.5114/aoms.2019.89835.

19. Lee EE, Hong S, Martin AS, Eyler LT, Jeste DV. Inflammation in schizophrenia: cytokine levels and their relationships to demographic and clinical variables. Am J Geriatr Psychiatry 2017; 25: 50-61.

20. Kuloglu M, Bayik Y, Unal A, Gecici O, Ustundag B. Serum IL-1 $\beta$, IL-2, IL-6, and IL-8 levels in schizophrenia subtypes. Bull Clin Psychopharmacol 2011; 21: 193-200.

21. Cannon TD, Chung Y, He G, et al. Progressive reduction in cortical thickness as psychosis develops: a multisite longitudinal neuroimaging study of youth at elevated clinical risk. Biol Psychiatry 2015; 77: 147-57.

22. Mondelli V, Cattaneo A, Murri MB, et al. Stress and inflammation reduce brain-derived neurotrophic factor expression in first-episode psychosis: a pathway to smaller hippocampal volume. J Clin Psychiatry 2011; 72: 1677-84.

23. DeLisi LE, Smith AB, Razi K, et al. Investigation of a candidate gene for schizophrenia on Xq13 previously associated with mental retardation and hypothyroidism. Am J Med Genet 2000; 96: 398-403.

24. Philibert RA, Sandhu HK, Hutton AM, et al. Population-based association analyses of the HOPA12bp polymorphism for schizophrenia and hypothyroidism. Am J Med Genet 2001; 105: 130-4.

25. Szcześniak G, Kozak-Nurczyk P, Dziemidok P. Does concomitance of Hashimoto's disease and type 1 diabetes affect diabetes control and development of its complications? Arch Med Sci 2019. DOI: https://doi.org/10.5114/ aoms.2019.89780.

26. Doria A, Zen M, Bettio S, Gatto M, et al. Autoinflammation and autoimmunity: bridging the divide. Autoimmun Rev 2012; 12: 22-30.

27. Bergen SE, O'Dushlaine CT, Ripke S, et al. Genome-wide association study in a Swedish population yields support for greater CNV and MHC involvement in schizophrenia compared with bipolar disorder. Mol Psychiatry 2012; 17: 880-6.

28. Lee SH, Byrne EM, Hultman CM, et al. New data and an old puzzle: the negative association between schizo- phrenia and rheumatoid arthritis. Int J Epidemiol 2015; 44: 1706-21.

29. Raychaudhuri S, Sandor C, Stahl EA, et al. Five amino acids in three HLA proteins explain most of the association between $\mathrm{MHC}$ and seropositive rheumatoid arthritis. Nat Genet 2012; 44: 291-6.

30. Yasunami M, Nakamura H, Agematsu K, et al. Identification of Disease-Promoting HLA Class I and Protective Class II Modifiers in Japanese Patients with Familial Mediterranean Fever. PLoS One 2015; 10: e0125938.

31. Saha S, Chant D, McGrath J. A systematic review of mortality in schizophrenia: is the differential mortality gap worsening over time? Arch Gen Psychiatry 2007; 64: 1123-31.

32. Twig G, Livneh A, Vivante A, et al. Mortality risk factors associated with familial Mediterranean fever among a cohort of 1.25 million adolescents. Ann Rheum Dis 2014; 73: 704-9. 